\title{
Inborn Errors of Metabolism That Cause Sudden Infant Death: A Systematic Review with Implications for Population Neonatal Screening Programmes
}

\author{
Willemijn J. van Rijt ${ }^{a}$ Geneviève D. Koolhaas ${ }^{c}$ Jolita Bekhof ${ }^{c}$ \\ M. Rebecca Heiner Fokkemab ${ }^{b}$ Tom J. de Koning ${ }^{a}$ Gepke Visser ${ }^{d}$ \\ Peter C.J.I. Schielen ${ }^{\mathrm{e}}$ Francjan J. van Spronsen ${ }^{\mathrm{a}}$ Terry G.J. Derks ${ }^{\mathrm{a}}$ \\ aSection of Metabolic Diseases, Beatrix Children's Hospital, and ' ${ }^{2}$ Laboratory of Metabolic Diseases, Department of \\ Laboratory Medicine, University Medical Center Groningen, University of Groningen, Groningen, 'Princess Amalia \\ Children's Clinic, Isala Hospital, Zwolle, d'Department of Metabolic Diseases, Wilhelmina Children's Hospital, UMCU, \\ Utrecht, and ' Laboratory of Infectious Diseases and Screening, National Institute of Public Health and Environment \\ (RIVM), Bilthoven, The Netherlands
}

\section{Key Words}

Neonatal screening · Inborn error of metabolism •

Mitochondrial fatty acid oxidation · Reye syndrome ·

Sudden infant death · Metabolic autopsy

\begin{abstract}
Background: Many inborn errors of metabolism (IEMs) may present as sudden infant death (SID). Nowadays, increasing numbers of patients with IEMs are identified pre-symptomatically by population neonatal bloodspot screening (NBS) programmes. However, some patients escape early detection because their symptoms and signs start before NBS test results become available, they even die even before the sample for NBS has been drawn or because there are IEMs which are not included in the NBS programmes. Objectives and Methods: This was a comprehensive systematic literature review to identify all IEMs associated with SID, including their treatability and detectability by NBS technologies. Reye syndrome (RS) was included in the search strategy because this
\end{abstract}

\section{KARGER}

E-Mail karger@karger.com www.karger.com/neo

\section{(C) 2016 The Author(s) \\ Published by S. Karger AG, Basel 1661-7800/16/1094-0297\$39.50/0}

This article is licensed under the Creative Commons Attribution NonCommercial-NoDerivatives 4.0 International License (CC BYNC-ND) (http://www.karger.com/Services/OpenAccessLicense) Usage and distribution for commercial purposes as well as any distribution of modified material requires written permission. condition can be considered a possible pre-stage of SID in a continuum of aggravating symptoms. Results: 43 IEMs were identified that were associated with SID and/or RS. Of these, (1) 26 can already present during the neonatal period, (2) treatment is available for at least 32 , and (3) 26 can currently be identified by the analysis of acylcarnitines and amino acids in dried bloodspots (DBS). Conclusion: We advocate an extensive analysis of amino acids and acylcarnitines in blood/ plasma/DBS and urine for all children who died suddenly and/or unexpectedly, including neonates in whom blood had not yet been drawn for the routine NBS test. The application of combined metabolite screening and DNA-sequencing techniques would facilitate fast identification and maximal diagnostic yield. This is important information for clinicians who need to maintain clinical awareness and decision-makers to improve population NBS programmes.

(C) 2016 The Author(s)

Published by S. Karger AG, Basel

Willemijn J. van Rijt and Geneviève D. Koolhaas contributed equally to this work.
Terry G.J. Derks, MD, PhD

Section of Metabolic Diseases, Beatrix Children's Hospital University Medical Center Groningen, University of Groningen PO Box 30 001, NL-9700 RB, Groningen (The Netherlands) E-Mail t.g.j.derks@umcg.nl 


\section{Introduction}

Many inborn errors of metabolism (IEMs) that cause cellular energy deficiency and/or intoxication are associated with sudden infant death (SID). Based on retrospective studies, approximately $0.9-6 \%$ of all SID cases involve IEMs [1-3]. Although these studies were subject to several forms of selection bias, they formed the rationale behind metabolic autopsy protocols for young children, which include analyses of amino acid and acylcarnitine profiles in plasma/urine [4].

Since the 1990s, tandem mass spectrometry (TMS) of dried blood spots (DBS) has been developed to perform high-throughput simultaneous quantitative analysis of different diagnostic metabolites in small amounts in biological samples [5]. As a consequence, in the last 2 decades, population neonatal bloodspot screening (NBS) programmes have expanded to include many IEMs. Patients with treatable IEMs can remain undetected by population NBS programmes for several reasons. In some IEMs, symptoms and signs including death may already occur before the NBS test results become available or even before blood for testing has been drawn, annulling the benefits of NBS [6-10]. This is especially relevant in areas where neonatal blood is collected relatively late, for instance, in the Netherlands (i.e. 72-168 h after birth) [11, 12]. Worldwide, across different areas, population NBS programmes differ with respect to the methodological aspects and the disorders screened.

Systematic studies on the percentage of IEMs in SID cases are required because, although rare, SID that is preventable due to the IEM concerned being treatable does still occur. Therefore, we performed this comprehensive systematic literature review to identify IEMs that (1) are associated with SID, (2) have clinical ascertainment during the neonatal period, (3) are treatable and (4) are detectable on TMS.

\section{Methods}

\section{Search Strategy}

A literature search for relevant references was performed according to the Cochrane Collaboration methodology. The CINAHL, Cochrane, PubMed and Embase public databases were searched using both $\mathrm{MeSH}$ terms and free text. A detailed presentation and assessment of the search strategy, including the Preferred Reporting Items for Systematic Review and Meta-Analysis Protocols (PRISMA-P) 2015 checklist, is presented in the online supplementary data (see www.karger.com/doi/10.1159/000443874 for all online suppl. material). Figure 1 presents the flow chart of the detailed search strategy together with the steps of the systematic review.

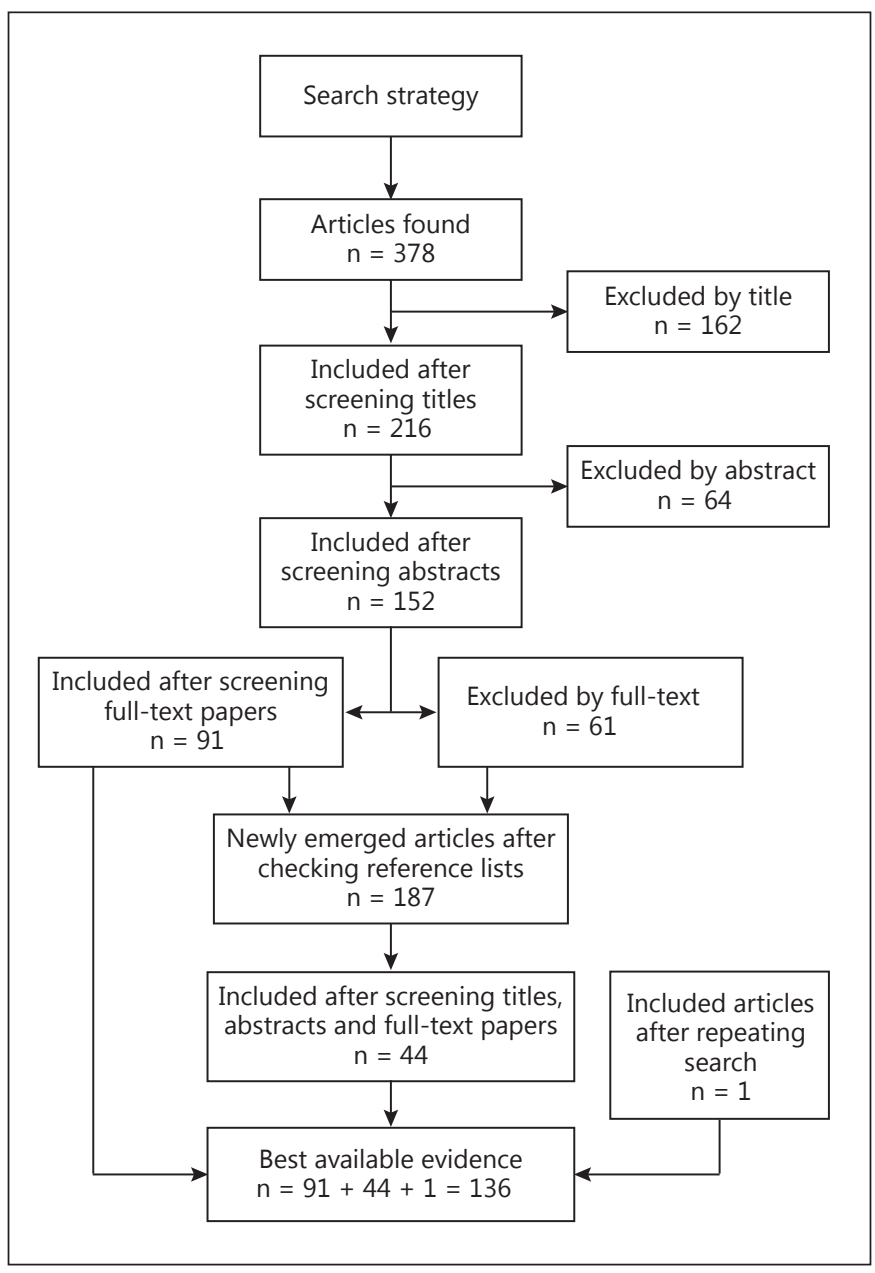

Fig. 1. Flow chart of detailed search strategy. CINAHL, Cochrane, PubMed and Embase were searched using both MeSH terms and free text: 'Metabolism, Inborn Errors' (MeSH) OR 'inborn errors of metabolism' OR 'mitochondrial fatty acid oxidation' AND 'Sudden Infant Death' (MeSH) OR 'sudden infant death' OR 'sudden infant death syndrome' OR 'unexpected death' OR 'sudden unexpected death of infant' OR 'Reye syndrome' (MeSH) AND 'Humans' (MeSH) AND 'Infant, Newborn, Child, Adolescent' (MeSH) OR 'newborn' OR 'infant' OR 'child'. The search was conducted on 15 February 2013. Due to the time that elapsed between the execution of the search and the completion of the paper, the search was repeated on 28 August 2015 to screen for possible extra IEMs. This led to the inclusion of only 1 more IEM associated with either SIDS and/or RS: dihydrolipoamide dehydrogenase deficiency (DLD deficiency; MIM No. 246900).

The following search terms were included to further optimize our search. The term 'mitochondrial fatty acid oxidation' was included because, based on previous studies and our personal expertise, this disease group has the highest incidence of IEMs associated with both SID [1-3] and NBS programmes $[6,7,10]$. SID is historically defined as occurring in the first year of life. We therefore expanded our search strategy, adding the term 'sudden unex- 
pected death of infant'. Originally, Reye syndrome (RS) was described as a non-inflammatory encephalopathy in childhood, associated with hepatic dysfunction [13]. Since the 1980s, it has been recognized as a presenting symptom of IEMs rather than an etiologic diagnosis [14]. We considered RS as a potential pre-stage of SID in a continuum of aggravating symptoms. We therefore also included the term 'Reye syndrome' in our search.

All reports published since 1990 were included, corresponding with the first publications about the availability of TMS and the general progressions made with molecular and enzymatic confirmatory testing in the field of IEMs. References published before 1990 were only included when available upon request. Two independent reviewers (G.K. and T.D.) performed title and abstract screenings. Consensus about inclusion was reached during regular meetings. Subsequently, 3 independent reviewers (W.v.R., G.K. and T.D.) screened the full-text articles of all selected references. The inclusion of a diagnosis as a cause of SID and/or RS was based on the presence of detailed patient data and a confirmed diagnosis in the full-text articles. Specific exclusion criteria were: (1) no detailed patient data reported, (2) a lack of accessibility of the articles, (3) confirmatory metabolite, molecular or enzymatic studies were inconclusive, (4) there had been a (possible) additional contributing cause of death, (5) RS patients were $>18$ years old and (6) the abstract and/or article was not available in either English or Dutch.

\section{Data Analysis}

All IEMs were classified according to the Society for the Study of Inborn Errors of Metabolism classification of IEMs [15]. Based on the included references, associations between confirmed diagnoses and SID and/or RS were documented (e.g. table 1: ' + ' in the SID column indicates that the particular IEM has been associated with SID in at least 1 of the corresponding references in online suppl. table 1). Neonatal clinical presentation was reported based on detailed patient data from the included references. Based on recent textbooks and the literature, the treatability [16] and detectability by TMS of a DBS [17-19] were documented, respectively.

\section{Results}

This systematic review included a total of 136 references. Table 1 presents the 43 IEMs associated with either SID and/or RS, concerning mostly disorders of mitochondrial fatty acid oxidation, the urea cycle and organic acidurias. References of all included articles are presented in online supplementary table 1 . Out of these 43 IEMs, 26 had presented already during the neonatal period, and 15 were found to be treatable and also detectable with TMS methodologies. In at least 32 of the IEMs, a specific dietary and/or pharmacological treatment is available in order to prevent clinical presentation. Identification by population NBS programmes by TMS analysis of amino acids and/or acylcarnitines in DBS is possible in 26 of the IEMs.

Inborn Errors of Metabolism That Cause Sudden Infant Death

\section{Discussion}

This unique systematic literature review identified at least 43 IEMs associated with SID and/or RS, 26 of which can already present during the neonatal period. At least 32 are considered as treatable disorders and 26 are currently detectable by TMS analysis of amino acids and/or acylcarnitines in DBS. The remaining 17 cannot be detected by current metabolite screening methods but require additional testing either by expanding the metabolic testing options or by means of genetic and/or enzymatic laboratory methods. Out of the 26 IEMs in which feasibility of clinical ascertainment within the neonatal period has been reported, at least 15 are treatable and are detectable by TMS analysis. This is important information to for the improvement of population NBS programmes because early detection and subsequent appropriate treatment can prevent clinical presentation and even death (table 1). Moreover, considering the results of our study, we propose that diagnostic (laboratory) protocols can be improved for children (including neonates) presenting with sudden/unexpected death.

There is no doubt that the expanded population NBS programmes have significantly improved the outcomes of many patients, but there is still a subset of patients that unfortunately escapes early identification [20]. In one group, this is because limited numbers of IEMs are included in NBS programmes. It is important to realize that population NBS programmes vary worldwide, and maybe even within countries. In another group, it is because the symptoms and signs present before the NBS test results become available or even before blood has been drawn. This is aggravated by the relatively late drawing of blood and/or follow-up after positive test results in some areas/ countries. In the Netherlands, the blood for the NBS test is collected between 72 and $168 \mathrm{~h}$ after birth $[11,12]$. In 2013, the response rate for the NBS programme was $99.35 \%$. Referral to a metabolic physician was initiated before day 8 in $62 \%$ of the positive neonates, but 441 out of 173,118 newborns died (etiology was not specified) before blood could be drawn [11]. The reports on population NBS programmes from Australia, the USA and Germany present patients with clinical ascertainment and sometimes even neonatal death before the NBS test results have become available (see: ${ }^{a}$ in table 1 ) $[6,7,10]$. In line with these reports, since the expansion of the NBS programme in our country (table 1), clinical symptoms and signs have often preceded the NBS test results, sometimes even leading to early death, in cases of very longchain acyl-CoA dehydrogenase deficiency, long-chain

Neonatology 2016;109:297-302 299 
Table 1. IEMs associated with SID and/or RS

Disorder

Neonatal RS

presentation

SID Treatable DBS Phenotype MIM No.

Amino acid and peptide metabolism

Urea cycle disorders

Carbamoylphosphate synthetase I deficiency

Ornithine transcarbamylase deficiency ${ }^{\mathrm{a}}$

Citrullinemia type $\mathrm{I}^{\mathrm{a}}$

Argininosuccinic aciduria

Organic acidurias

Glutaric aciduria type $\mathrm{I}^{\mathrm{a}, \mathrm{b}}$

Propionic aciduria ${ }^{\mathrm{a}, \mathrm{c}}$

Methylmalonic aciduria ${ }^{\text {a, }}$

Isovaleric aciduria $^{\text {a, b }}$

Methylglutaconic aciduria, type I

Methylglutaconic aciduria, type II (Barth syndrome)

3-Hydroxy-3-methylglutaric aciduria

Alpha-methylacetoacetic aciduria ${ }^{c}$

L-2-hydroxyglutaric aciduria

Disorders of the metabolism of branched-chain amino acids not classified as organic acidurias

Dihydrolipoamide dehydrogenase deficiency ${ }^{1}$

Disorders of phenylalanine or tyrosine metabolism

Tyrosinemia type $\mathrm{I}^{\mathrm{a}, \mathrm{b}}$

Disorders of serine, glycine glycerate metabolism

Nonketotic hyperglycinemia ${ }^{a}$

Disorders of amino acid transport

Lysinuric protein intolerance

$\begin{array}{llllll}+ & + & + & + & +{ }^{\mathrm{d}} & 237300 \\ + & + & + & + & +{ }^{\mathrm{d}} & 311250 \\ + & + & + & + & +{ }^{\mathrm{d}} & 215700 \\ - & - & + & + & +^{\mathrm{d}} & 207900 \\ & & & & & \\ - & - & + & + & +{ }^{\mathrm{d}} & 231670 \\ + & + & - & + & +{ }^{\mathrm{d}} & 232000 \\ + & + & + & + & +{ }^{\mathrm{d}} & 251000 \\ - & - & + & + & +{ }^{\mathrm{d}} & 243500 \\ - & + & - & + & +{ }^{\mathrm{d}} & 250950 \\ + & + & + & + & - & 302060 \\ + & + & - & + & +{ }^{\mathrm{d}} & 246450 \\ - & + & - & + & +{ }^{\mathrm{d}} & 203750 \\ - & - & + & - & - & 236792\end{array}$

Carbohydrate metabolism

Disorders of fructose metabolism

Hereditary fructose intolerance

Disorders of glycerol metabolism

Glycerol kinase deficiency

Disorders of gluconeogenesis

Fructose-1,6-biphosphatase deficiency

Phosphoenolpyruvate carboxykinase deficiency

Glycogen storage disorders

Glycogen storage disease type Ia (von Gierke disease)

Glycogen storage disease type Ib

Glycogen storage disease type II (Pompe disease)

$\begin{array}{cccccc}+ & + & - & - & - & 246900 \\ + & + & + & + & +{ }^{\mathrm{d}} & 276700 \\ + & - & + & +/- & +{ }^{\mathrm{d}} & 238300 \\ - & - & + & + & - & 222700\end{array}$

Fatty acid and ketone body metabolism

Disorders of carnitine transport and the carnitine cycle

Carnitine transporter deficiency ${ }^{\mathrm{c}}$

Carnitine palmitoyltransferase I deficiency ${ }^{c}$

Carnitine acylcarnitine translocase deficiency ${ }^{c}$

Carnitine palmitoyltransferase II deficiency ${ }^{c}$

Disorders of mitochondrial fatty acid oxidation

Very long-chain acyl-CoA dehydrogenase deficiency ${ }^{\mathrm{a}, \mathrm{b}}$

Mitochondrial trifunctional protein deficiency

Isolated deficiency of long-chain 3-hydroxylacyl-CoA dehydrogenase ${ }^{\mathrm{b}}$

Medium-chain acyl-CoA dehydrogenase deficiency ${ }^{\mathrm{a}, \mathrm{b}}$

Medium-chain 3-ketoacyl-CoA thiolase deficiency ${ }^{2}$

3-Alpha-hydroxylacyl-CoA dehydrogenase deficiency

Multiple acyl-CoA dehydrogenase deficiency ${ }^{\mathrm{a}}$

$\begin{array}{llllll}- & + & - & + & - & 229600 \\ + & + & - & + & - & 307030 \\ + & + & - & + & - & 229700 \\ - & + & + & - & - & 261650 \\ + & + & + & + & - & 232200 \\ - & - & + & + & - & 232220 \\ - & - & + & + & +^{\mathrm{e}} & 232300\end{array}$

229600 
Table 2 (continued)

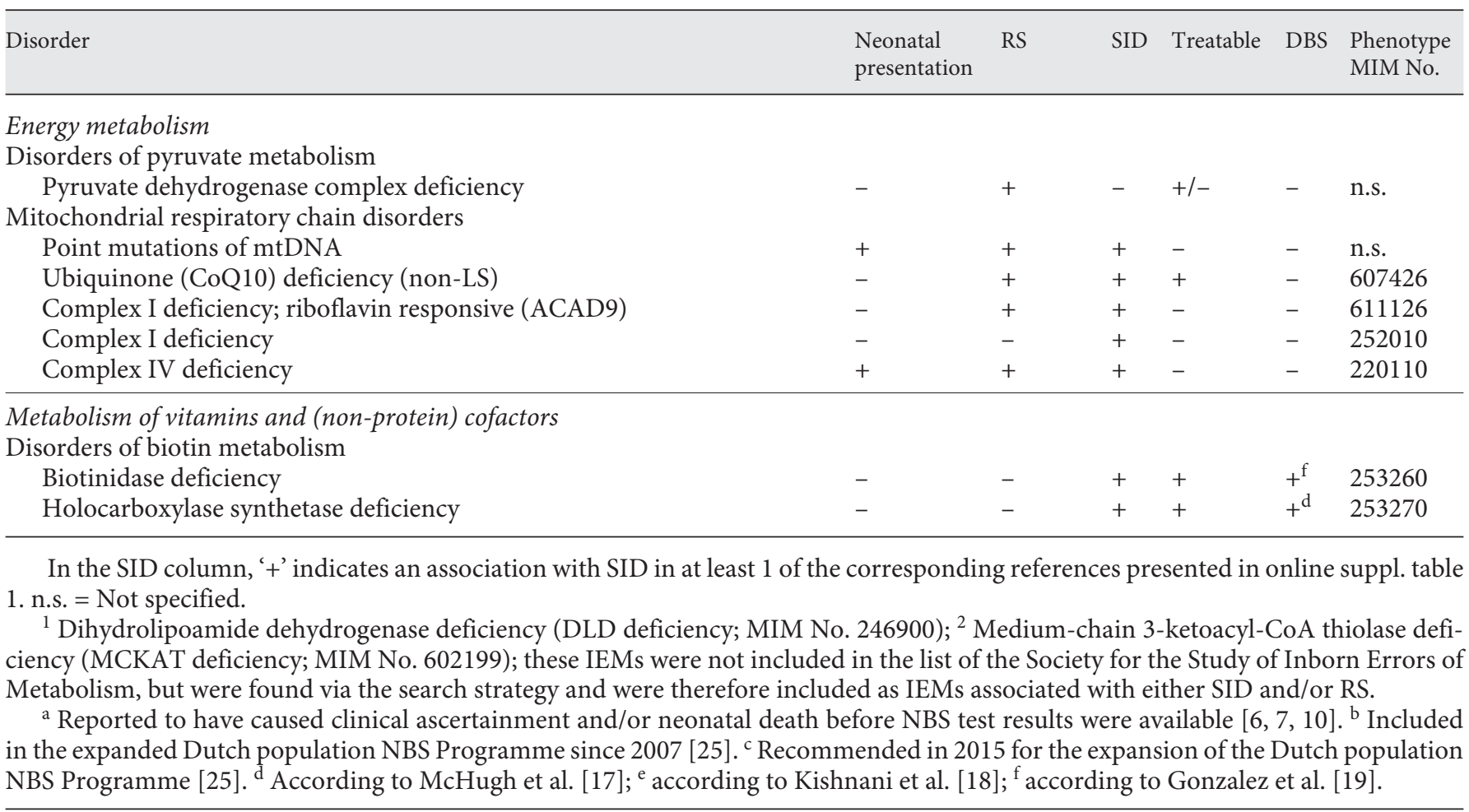

3-hydroxyacyl-CoA dehydrogenase deficiency/mitochondrial trifunctional protein deficiency, mediumchain acyl-CoA dehydrogenase deficiency, maple syrup urine disease and galactosemia (unpubl. data). Patients may also escape early identification due to false-negative NBS results (e.g. patients with carnitine transporter deficiency or very long-chain acyl-CoA dehydrogenase deficiency $[21,22])$ or for analytical reasons, which is of concern for patients with carnitine palmitoyltransferase 2 deficiency [23]. These examples stir up the debate on whether the NBS test should be performed earlier in life and/or at 2 different time points.

The general view on 'the metabolic autopsy' originated from case studies and small retrospective cohort studies that introduced bias [4]. It is generally recognized that low incidences and aspecific symptoms and signs cause an under-diagnosis of IEMs [9]. Our study strengthens the rationale that, despite a low incidence of individual IEMs, the neonates who died deserved at least a TMS analysis of amino acids and acylcarnitines in a DBS, when feasible. For most of the disorders listed in table 1, the associated recurrence rate for affected families is at least $25 \%$.

Inborn Errors of Metabolism That Cause Sudden Infant Death
Several methodological issues in this study should be mentioned. First, the retrospective design of many of the cohort studies and case studies included might have introduced both a publication bias and a data availability bias as (1) the reports do not always describe detailed patient data and (2) obviously not all SID cases due to IEMs get reported in the literature. Second, there are many factors including aspecific symptoms that lead to under-diagnosis of IEMs in neonates [9]. Third, despite our extensive and detailed search strategy, we cannot exclude the possibility that a few references were missed. This was emphasized by the fact that, after including the full-text articles in the first round $(n=91)$, new references still emerged via the reference lists of excluded and included full-text articles. In order to optimize the search strategy, we conducted a second $(\mathrm{n}=44)$ and third $(\mathrm{n}=1)$ screening round. Fourth, in the medical literature, the definition of SID is not always consistently applied with regard to age range and clinical symptoms and signs. In an attempt to overcome this, we added the term 'sudden unexpected death of infant' to our search strategy. Last, some included IEMs exemplify only one protein deficiency in a large metabolic pathway involving many enzymes and transporters that could potentially create a similar clinical pic- 
ture. Therefore, we believe, based on our systematic review, that the IEMs included in table 1 should be considered as the minimal number of IEMs associated with SID and/or RS. Despite expanding NBS programmes, clinical awareness needs to remain high amongst neonatologists and paediatricians because many IEMs have not yet been implemented in NBS programmes. It is possible that early recognition of clinical presentations and subsequent diagnostic testing could prevent fatal outcomes [24].

In summary, our systematic review identified the IEMs that are associated with RS and SID, a significant proportion of which are treatable disorders. In our opinion, the analysis of amino acids and acylcarnitines in blood/plas$\mathrm{ma} / \mathrm{DBS}$ and urine should be part of post-mortem diagnostic protocol, next to the isolation of DNA and, prefer- entially, material for functional tests such as the analysis of cultured skin fibroblasts. The combination of metabolite screening and DNA-sequencing techniques would harbor the best of both methods, i.e. fast identification and a high diagnostic yield.

\section{Acknowledgements}

The authors would like to thank Mirell H.G. Papenhuijzen for her assistance in performing the search.

\section{Disclosure Statement}

The authors have nothing to declare.

\section{References}

-1 Boles RG, Buck EA, Blitzer MG, Platt MS, Cowan TM, Martin SK, Yoon H, Madsen JA, Reyes-Mugica M, Rinaldo P: Retrospective biochemical screening of fatty acid oxidation disorders in postmortem livers of 418 cases of sudden death in the first year of life. J Pediatr 1998;132:924-933.

-2 Chace DH, DiPerna JC, Mitchell BL, Sgroi B, Hofman LF, Naylor EW: Electrospray tandem mass spectrometry for analysis of acylcarnitines in dried postmortem blood specimens collected at autopsy from infants with unexplained cause of death. Clin Chem 2001;47: 1166-1182.

3 Green A, Preece MA, Hardy D: More on the metabolic autopsy. Clin Chem 2002;48:964965.

4 Ernst LM, Sondheimer N, Deardorff MA, Bennett MJ, Pawel BR: The value of the metabolic autopsy in the pediatric hospital setting. J Pediatr 2006;148:779-783.

5 Millington DS, Kodo N, Norwood DL, Roe CR: Tandem mass spectrometry: a new method for acylcarnitine profiling with potential for neonatal screening for inborn errors of metabolism. J Inherit Metab Dis 1990;13:321-324.

6 Wilcken B, Wiley V, Hammond J, Carpenter $\mathrm{K}$ : Screening newborns for inborn errors of metabolism by tandem mass spectrometry. $\mathrm{N}$ Engl J Med 2003;348:2304-2312.

-7 Frazier DM, Millington DS, McCandless SE, Koeberl DD, Weavil SD, Chaing SH, Muenzer $\mathrm{J}$ : The tandem mass spectrometry newborn screening experience in North Carolina: 19972005. J Inherit Metab Dis 2006;29:76-85.

-8 Yusuf K, Jirapradittha J, Amin HJ, Yu W, Hasan SU: Neonatal ventricular tachyarrhythmias in medium chain acyl-CoA dehydrogenase deficiency. Neonatology 2010;98:260-264.

9 Derks TG: Sweet and sour aspects of mediumchain acyl CoA dehydrogenase deficiency. Commentary on K. Yusuf et al.: Neonatal ventricular tachyarrhythmias in medium chain
acyl-CoA dehydrogenase deficiency (Neonatology 2010;98:260-264). Neonatology 2010; 98:265-267.

10 Lindner M, Gramer G, Haege G, Fang-Hoffmann J, Schwab KO, Tacke U, Trefz FK, Mengel E, Wendel U, Leichsenring M, Burgard P, Hoffmann GF: Efficacy and outcome of expanded newborn screening for metabolic diseases - report of 10 years from South-West Germany. Orphanet J Rare Dis 2011;6:441172.

11 Rijpstra A, Schonbeck Y, Verkerk PH: Evaluatie van de Neonatale Hielprikscreening bij Kinderen Geboren in 2013. Leiden, TNO, 2015.

12 Centrum voor Bevolkingsonderzoek: Draaiboek Neonatale Hielprikscreening. Bilthoven, Rijksinstituut voor Volksgezondheid en $\mathrm{Mi}$ lieu, 2015.

13 Reye RD, Morgan G, Baral J: Encephalopathy and fatty degeneration of the viscera. A disease entity in childhood. Lancet 1963;2:749-752.

14 Orlowski JP: Whatever happened to Reye's syndrome? Did it ever really exist? Crit Care Med 1999;27:1582-1587.

15 Society for the Study of Inborn Errors of Metabolism: Classification of Inborn Errors of Metabolism 2011.

16 Saudubray JM, van den Berghe G, Walter JH: Inborn Metabolic Diseases: Diagnosis and Treatment, ed 5 (revised). Heidelberg, Springer, 2011.

17 McHugh D, Cameron CA, Abdenur JE, et al: Clinical validation of cutoff target ranges in newborn screening of metabolic disorders by tandem mass spectrometry: a worldwide collaborative project. Genet Med 2011;13:230254.

18 Kishnani PS, Steiner RD, Bali D, Berger K, Byrne BJ, Case LE, Crowley JF, Downs S, Howell RR, Kravitz RM, Mackey J, Marsden D, Martins AM, Millington DS, Nicolino M, O'Grady G, Patterson MC, Rapoport DM, Slonim A, Spencer CT, Tifft CJ, Watson MS;
ACMG Work Group on Management of Pompe Disease: Pompe disease diagnosis and management guideline. Genet Med 2006;8: 267-288.

19 Gonzalez EC, Marrero N, Frometa A, Herrera D, Castells E, Perez PL: Qualitative colorimetric ultramicroassay for the detection of biotinidase deficiency in newborns. Clin Chim Acta 2006;369:35-39.

20 Marsden D, Larson C, Levy HL: Newborn screening for metabolic disorders. J Pediatr 2006;148:577-584.

21 Lund AM, Hougaard DM, Simonsen H, Andresen BS, Christensen M, Duno M, Skogstrand K, Olsen RK, Jensen UG, Cohen A, Larsen N, Saugmann-Jensen P, Gregersen N, Brandt NJ, Christensen E, Skovby F, Norgaard-Pedersen B: Biochemical screening of 504,049 newborns in Denmark, the Faroe Islands and Greenland - experience and development of a routine program for expanded newborn screening. Mol Genet Metab 2012; 107:281-293.

22 Ficicioglu C, Coughlin CR 2nd, Bennett MJ, Yudkoff M: Very long-chain acyl-CoA dehydrogenase deficiency in a patient with normal newborn screening by tandem mass spectrometry. J Pediatr 2010;156:492-494.

23 de Sain-van der Velden MG, Diekman EF, Jans JJ, van der Ham M, Prinsen BH, Visser G, Verhoeven-Duif NM: Differences between acylcarnitine profiles in plasma and bloodspots. Mol Genet Metab 2013;110:116-121.

24 Derks TG, Jakobs H, Gerding A, Niezen-Koning KE, Reijngoud DJ, Smit GP: Deficiency of the fatty-acid oxidising enzyme mediumchain acyl-CoA dehydrogenase (MCAD) in an adult, detected during a neonatal screening programme. Ned Tijdschr Geneeskd 2004; 148:2185-2190.

25 Health Council of the Netherlands: Neonatal screening: new recommendations 2015; 2015/08. 